\title{
Laparoscopic repeat hepatectomy for recurrent hepatocellular carcinoma from segment 1 to 8: Is it a feasible treatment strategy?
}

\author{
Theo Genesis TAGAYTAY ${ }^{1,2}$, Dai Hoon HAN', Gi Hong CHOI*, ${ }^{* 1}$ Kyung Sik KIM', Jin Sub CHOI' \\ 'Division of Hepatobiliary and Pancreatic Surgery, Department of Surgery, Yonsei University College of Medicine, Seoul, Korea \\ ${ }^{2}$ Department of General Surgery, Vicente Sotto Memorial Medical Center, Cebu, Philippines
}

Introduction: Laparoscopic liver resection for hepatocellular carcinoma (HCC) is becoming the primary option for expert surgeons and high volume centers. However, repeat liver resection for recurrent HCC is still not yet established. This study aims to determine the surgical outcomes with laparoscopic repeat liver resection (LRLR) vs. open repeat liver resection (ORLR) for recurrent HCC.

Methods: Fifty cases of repeat liver resection was done in our center since January 2009 to November 2020. The repeat surgery was performed under LRLR group for 23 patients and ORLR group for 27 patients. The surgical outcomes were studied retrospectively. Results: We had $2(8.6 \%)$ open conversion secondary to bleeding and severe adhesion from previous surgery. Most of the LRLR, 17 (73.9\%) patients had primary laparoscopic liver resection. The LRLR had shorter operative time (mean 159.74 vs. 250.19 minutes; $p \leq$ 0.001 ), lesser intraoperative blood loss (mean 185.65 vs. $385.56 \mathrm{~mL} ; p=0.037$ ), lower morbidity ( $8.6 \%$ vs. $62.9 \% ; p \leq 0.001)$ and shorter hospital stay (mean 5.83 vs. 9.26 days; $p \leq 0.001$ ) compared with ORLR. There was no significant difference in the R0 resection and surgical margin between the two groups.

Conclusions: LRLR is feasible and safe for highly selective patients. With the expert surgeons and in high volume centers, LRLR offers betters perioperative outcomes without compromising oncologic benefits compared with ORLR. 